Baltic Astronomy, vol. 19, 157-167, 2010

\title{
CHEMICAL COMPOSITION OF THE RS CVn-TYPE STAR 29 DRACONIS
}

\author{
G. Barisevičius, ${ }^{1}$ G. Tautvaišienè, ${ }^{1}$ S. Berdyugina, ${ }^{2}$ Y. Chorniy ${ }^{1}$ and I. Ilyin ${ }^{3}$ \\ 1 Institute of Theoretical Physics and Astronomy, Vilnius University, \\ Goštauto 12, Vilnius, LT-01108, Lithuania \\ 2 Kiepenheuer Institut für Sonnenphysik, Schöneckstr. 6, D-79104 Freiburg, \\ Germany \\ 3 Astrophysical Institute Potsdam, An der Sternwarte 16, Potsdam D-14482, \\ Germany
}

Received 2010 October 2; accepted 2010 November 5

\begin{abstract}
Photospheric parameters and chemical composition are determined for the single-lined chromospherically active RS CVn-type star 29 Draconis (HD 160538). From the high resolution spectra obtained on the Nordic Optical Telescope, abundances of 22 chemical elements, including the key elements such as ${ }^{12} \mathrm{C},{ }^{13} \mathrm{C}, \mathrm{N}$ and $\mathrm{O}$, were investigated. The differential line analysis with the MARCS model atmospheres gives $T_{\text {eff }}=4720 \mathrm{~K}, \log g=2.5,[\mathrm{Fe} / \mathrm{H}]=-0.20$, $[\mathrm{C} / \mathrm{Fe}]=-0.14,[\mathrm{~N} / \mathrm{Fe}]=0.08,[\mathrm{O} / \mathrm{Fe}]=-0.04, \mathrm{C} / \mathrm{N}=2.40,{ }^{12} \mathrm{C} /{ }^{13} \mathrm{C}=16$. The low value of the ${ }^{12} \mathrm{C} /{ }^{13} \mathrm{C}$ ratio gives a hint that extra mixing processes in low-mass chromospherically active stars may start earlier than the theory of stellar evolution predicts.
\end{abstract}

Key words: stars: RS CVn binaries, abundances - stars: individual (29 Draconis $=$ HD 160538))

\section{INTRODUCTION}

In this work we continue a detailed study of photospheric abundances in RS CVn stars (Tautvaišienè et al. 2010, hereafter Paper I) with the aim to get new data on chemical evolution of the photospheric and coronal abundance patterns and mixing processes in chromospherically active stars. Our aim is to investigate abundances of more than 20 chemical elements, including ${ }^{12} \mathrm{C},{ }^{13} \mathrm{C}, \mathrm{N}, \mathrm{O}$ and other mixing sensitive species. We plan to investigate correlations between the abundance alterations of chemical elements in star atmospheres and their physical macro parameters, such as the speed of rotation and the magnetic field.

Here we present the results of the analysis for the RS CVn binary 29 Draconis (HD 160538) consisting of a K0 III star $(V \approx 6.64 \mathrm{mag})$ and a white dwarf. Since the primary dominates the spectrum, the photospheric abundances can be relatively well determined, and thus the system is a good candidate for studying the coronal-to-photospheric abundance patterns.

Hall et al. (1982) were the first to suggest that 29 Draconis is a RS CVn binary. Its late spectral type (K0 III according to Bidelman 1954 or K2 III according to Roman 1955), the CaII H \& K emission in its spectrum (Bidelman 1954), radial 
velocity values by Abt \& Biggs (1972) and photometric variability reaching $\Delta V=$ $0.12 \mathrm{mag}$, as well as a rotation period of 31.5 days found by Hall et al., allowed the authors to conclude that 29 Dra is a RS CVn binary.

Ultraviolet spectra of 29 Dra were observed by Fekel \& Simon (1985). It was found that the absorption lines are slightly broadened by stellar rotation with $v \sin i=8 \pm 2 \mathrm{kms}^{-1}$. The $\mathrm{H} \alpha$ absorption line was weak, as if filled in by emission, and variable. In five observations obtained over a period of four months, the equivalent width ranged from 0.1 to $0.8 \AA$. Mg II $h$ and $k$ lines were in emission. The study by Fekel \& Simon has revealed a white dwarf companion of 29 Dra, whose ultraviolet spectrum is matched best to the energy distribution of a $T_{\text {eff }}=30000 \mathrm{~K}$ and $\log g=8$ model atmosphere. The mass and radius of the white dwarf were found to be $0.55 M_{\odot}$ and $0.012 R_{\odot}$, respectively. A radius of the primary component was evaluated to be more than $5 R_{\odot}$. Fekel et al. (1993) derived $v \sin i=7.2 \mathrm{~km} \mathrm{~s}^{-1}$, the orbital period 903.8 days and the parameters for the companion star. They suspected that 29 Dra can be a barium-like star.

However, high-resolution spectral analyses by Berdyugina (1994) and Začs et al. (1997) have ruled out the hypothesis that 29 Dra is a barium-like star.

In further investigations of 29 Dra, Fekel (1997) determined a more accurate value of the radius of the primary component, $4.2 R_{\odot}$, and the projected rotational velocity $v \sin i=6.7 \mathrm{~km} \mathrm{~s}^{-1}$, which was used in our work.

A long-term photometry of 29 Dra, which lasted about 28 years, was recently reported by Zboril \& Messina (2009). They found that this star has three magnetic cycles $(20.3,11.1$ and $7.6 \mathrm{yr})$. Modeling of the light curve indicated that the spots cover from 4 to $10 \%$ of total stellar surface. The photometric period was found to be variable in the range $26.4-31.8 \mathrm{~d}$, suggesting differential rotation.

In our recent investigation of $\lambda$ And (Paper I), we found a low ratio of ${ }^{12} \mathrm{C} /{ }^{13} \mathrm{C}$ $=14$, which is lower than is predicted by the first dredge-up theory for a firstascent giant with the parameters of $\lambda$ And. Masses and luminosities of 29 Dra and $\lambda$ And are very similar. In this work, it was interesting to check a hint that extra mixing processes in low-mass chromospherically active fast rotating stars may start earlier than in non-active stars, i.e., below the bump of the luminosity function of red giant branch.

\section{OBSERVATIONS AND THE METHOD OF ANALYSIS}

The spectra of 29 Dra were obtained in 1999 August on the 2.56 m Nordic Optical Telescope using the SOFIN echelle spectrograph with the optical camera, which provided a spectral resolving power of $R \approx 80000$, for 26 spectral orders, each of $\sim 40 \AA$, in the spectral region from 5000 to $8300 \AA$. The details of spectral reductions were presented in Paper I.

In the spectra we selected 148 atomic lines for the measurement of equivalent widths (Table 1) and 17 lines for the comparison with synthetic spectra. The spectra were analyzed using a differential model atmosphere technique described in Paper I. Here we present only some details.

\subsection{Atmospheric parameters}

Initially, the effective temperature, $T_{\text {eff }}$, of 29 Dra was derived and averaged from the intrinsic color indices $(B-V)_{0}$ and $(b-y)_{0}$ using the corrected calibrations by Alonso et al. (1999). The values of color indices $B-V=1.043$ and $b-y=$ 
Table 1. Measured equivalent widths of lines, $E W$, in the spectrum of 29 Dra.

\begin{tabular}{|c|c|c|c|c|c|c|c|c|}
\hline Element & $\lambda(\AA)$ & $E W(\mathrm{~m} \AA)$ & Element & $\lambda(\AA)$ & $E W(\mathrm{~m} \AA)$ & Element & $\lambda(\AA)$ & $E W(\mathrm{~m} \AA)$ \\
\hline \multirow[t]{8}{*}{ Si I } & 5517.55 & 15.1 & \multirow{13}{*}{ Cr I } & 6266.30 & 47.6 & & 6786.86 & 49.0 \\
\hline & 5645.60 & 55.0 & & 6274.66 & 75.4 & & 6793.27 & 30.9 \\
\hline & 5665.55 & 55.1 & & 6285.16 & 83.1 & & 6839.83 & 83.2 \\
\hline & 5793.08 & 50.5 & & 6292.82 & 86.8 & & 6842.69 & 59.6 \\
\hline & 5948.54 & 92.1 & & & & & 6843.65 & 86.0 \\
\hline & 6131.85 & 28.8 & & 5712.78 & 49.6 & & 6851.64 & 34.4 \\
\hline & 7003.57 & 51.3 & & 5783.87 & 75.4 & & 6857.25 & 45.8 \\
\hline & & & & 5784.97 & 58.3 & & 6858.15 & 73.9 \\
\hline \multirow[t]{5}{*}{ Ca I } & 5260.38 & 76.3 & & 5787.92 & 82.8 & & 6862.49 & 50.5 \\
\hline & 5867.57 & 60.0 & & 6661.08 & 27.4 & & 7461.53 & 75.0 \\
\hline & 6455.60 & 107.7 & & 6979.80 & 71.3 & & & \\
\hline & 6798.47 & 28.1 & & 6980.91 & 29.6 & Fe II & 5132.68 & 37.4 \\
\hline & & & & & & & 5264.81 & 49.2 \\
\hline \multirow[t]{6}{*}{ Sc II } & 5526.81 & 111.8 & \multirow[t]{6}{*}{$\mathrm{Fe} I$} & 5395.22 & 39.9 & & 6113.33 & 17.5 \\
\hline & 5640.98 & 77.4 & & 5406.78 & 59.7 & & 6369.46 & 25.0 \\
\hline & 5667.14 & 62.5 & & 5522.45 & 67.4 & & 6456.39 & 64.7 \\
\hline & 6279.75 & 57.3 & & 5577.03 & 21.0 & & 7711.72 & 44.8 \\
\hline & 6300.69 & 15.9 & & 5579.35 & 24.4 & & & \\
\hline & & & & 5607.67 & 36.7 & Co I & 5530.78 & 71.2 \\
\hline \multirow[t]{19}{*}{ Ti I } & 5648.58 & 45.4 & & 5608.98 & 29.7 & & 5590.71 & 60.6 \\
\hline & 5662.16 & 69.3 & & 5651.48 & 35.4 & & 5647.23 & 51.7 \\
\hline & 5716.45 & 32.5 & & 5652.33 & 48.7 & & 6117.00 & 41.8 \\
\hline & 5739.48 & 42.5 & & 5653.86 & 62.3 & & 6188.98 & 59.1 \\
\hline & 5880.27 & 54.6 & & 5679.03 & 81.4 & & 6455.00 & 37.7 \\
\hline & 5899.30 & 104.6 & & 5720.90 & 31.1 & & 6595.86 & 17.9 \\
\hline & 5903.31 & 46.5 & & 5732.30 & 24.0 & & 6678.82 & 38.8 \\
\hline & 5941.76 & 73.0 & & 5738.24 & 31.0 & & & \\
\hline & 5953.17 & 88.1 & & 5741.86 & 54.0 & $\mathrm{Ni} I$ & 5578.73 & 98.1 \\
\hline & 5965.83 & 84.5 & & 5784.67 & 59.9 & & 5587.87 & 100.2 \\
\hline & 6064.63 & 62.4 & & 5793.92 & 59.5 & & 5589.37 & 48.4 \\
\hline & 6098.66 & 23.3 & & 5806.73 & 75.0 & & 5593.75 & 61.0 \\
\hline & 6121.00 & 29.3 & & 5807.79 & 28.1 & & 5643.09 & 27.8 \\
\hline & 6126.22 & 88.6 & & 5809.22 & 81.5 & & 5748.35 & 77.6 \\
\hline & 6220.49 & 45.4 & & 5811.92 & 25.9 & & 5805.22 & 56.7 \\
\hline & 6303.77 & 54.5 & & 5814.82 & 44.0 & & 6053.68 & 32.0 \\
\hline & 6599.11 & 65.7 & & 6027.06 & 89.0 & & 6108.12 & 113.8 \\
\hline & 6861.45 & 39.7 & & 6034.04 & 19.8 & & 6111.08 & 50.7 \\
\hline & & & & 6035.35 & 15.2 & & 6128.98 & 71.5 \\
\hline \multirow[t]{15}{*}{ VI } & 5604.96 & 34.8 & & 6054.07 & 22.1 & & 6130.14 & 33.8 \\
\hline & 5646.11 & 45.3 & & 6056.01 & 90.0 & & 6204.60 & 38.4 \\
\hline & 5657.45 & 52.6 & & 6098.25 & 31.6 & & 6378.25 & 49.4 \\
\hline & 5668.37 & 52.9 & & 6105.13 & 22.8 & & 6482.80 & 85.6 \\
\hline & 5670.86 & 85.3 & & 6120.24 & 45.8 & & 6586.32 & 91.3 \\
\hline & 5727.66 & 68.1 & & 6187.99 & 77.1 & & 6598.60 & 38.3 \\
\hline & 5737.07 & 76.4 & & 6200.32 & 123.8 & & 6635.13 & 36.2 \\
\hline & 5743.43 & 59.1 & & 6226.74 & 56.8 & & 6767.78 & 140.1 \\
\hline & 6039.74 & 72.9 & & 6229.23 & 83.6 & & 6772.32 & 75.3 \\
\hline & 6058.18 & 36.1 & & 6270.23 & 95.5 & & 6842.03 & 48.0 \\
\hline & 6111.65 & 76.3 & & 6380.75 & 79.8 & & 7001.55 & 46.9 \\
\hline & 6119.53 & 87.2 & & 6392.54 & 60.0 & & 7062.97 & 50.9 \\
\hline & 6135.37 & 70.6 & & 6574.21 & 99.0 & & 7715.59 & 74.9 \\
\hline & 6224.50 & 68.8 & & 6581.21 & 78.8 & & & \\
\hline & 6233.19 & 65.6 & & 6646.97 & 43.4 & & & \\
\hline
\end{tabular}


0.664 were taken from van Leeuwen et al. (2007) and Hauck \& Mermilliod (1998), respectively. A small dereddening correction of $E_{B-V}=0.02$, estimated using the Hakkila et al. (1997) software, was taken into account.

The agreement between the temperatures deduced from the two color indices was quite good, with the difference $70 \mathrm{~K}$ only. No obvious trend of the Fe I abundances with the excitation potential was found (Figure 1).

The surface gravity $\log g$ was found by adjusting the model gravity to yield the same iron abundance from the Fe I and Fe II lines. The microturbulent velocity $v_{\mathrm{t}}$ value corresponding to a minimal line-to-line Fe I abundance scattering was chosen as a correct value. Consequently, $[\mathrm{Fe} / \mathrm{H}]$ values do not depend on the equivalent widths of lines (Figure 2).

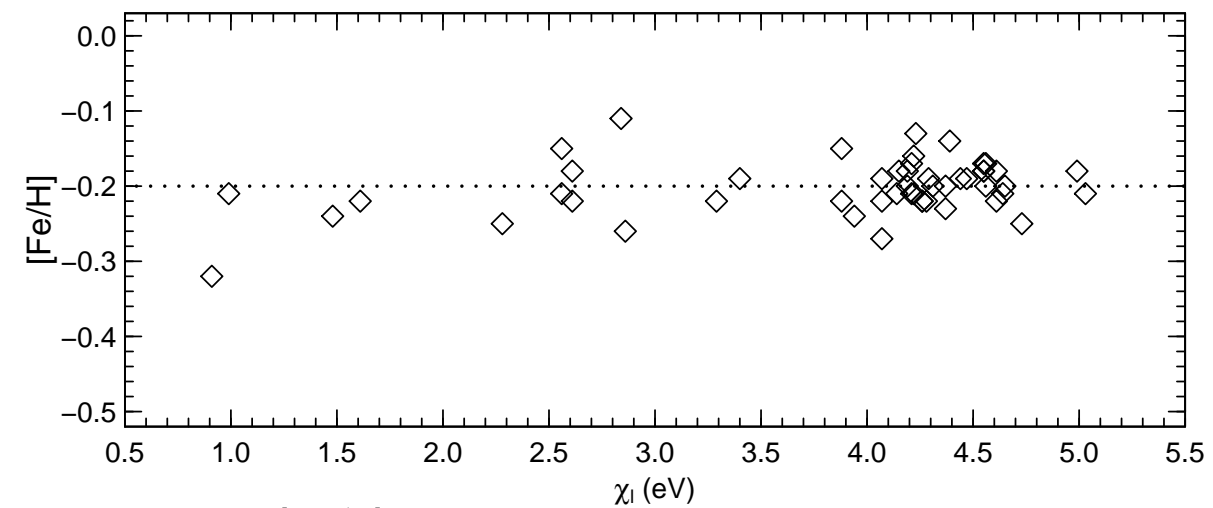

Fig. 1. The $[\mathrm{FeI} / \mathrm{H}]$ abundance values versus the lower excitation potential $\chi_{1}$. The mean abundance $([\mathrm{Fe} \mathrm{I} / \mathrm{H}]=-0.20 \mathrm{dex})$ is shown as a dotted line.

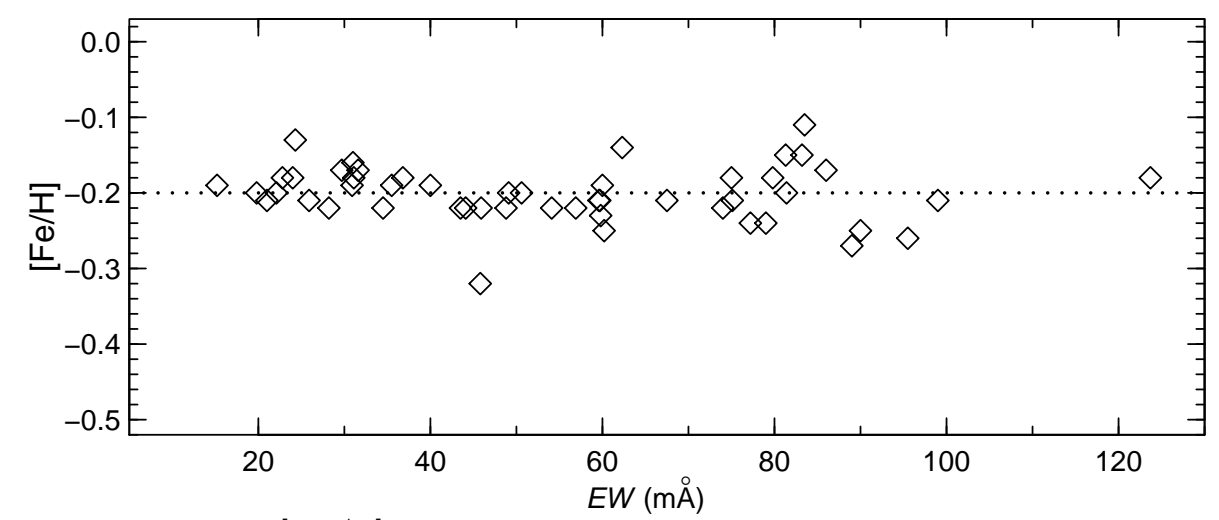

Fig. 2. The $[\mathrm{Fe} / \mathrm{H}]$ abundance values versus the equivalent widths. The mean abundance $([\mathrm{Fe} \mathrm{I} / \mathrm{H}]=-0.20 \mathrm{dex})$ is shown as a dotted line.

\subsection{Mass determination}

The mass of 29 Dra was evaluated from its effective temperature, luminosity and the isochrones from Girardi et al. (2000). The luminosity $\log \left(L / L_{\odot}\right)=1.44$ was calculated from the Hipparcos parallax $\pi=9.66$ mas (van Leeuwen 2007) and $V=6.64 \mathrm{mag}$, the bolometric correction calculated according to Alonso et al. 
(1999), and the above mentioned $E_{B-V}=0.02$. The mass of 29 Dra $\sim 1.2 M_{\odot}$ was found.

\subsection{Spectrum syntheses}

The method of synthetic spectra was used to determine carbon abundance from the $\mathrm{C}_{2}$ line at $5135.5 \AA$. The interval $7980-8130 \AA$, containing strong ${ }^{12} \mathrm{C}^{14} \mathrm{~N}$ and ${ }^{13} \mathrm{C}^{14} \mathrm{~N}$ features, was used for the nitrogen abundance and ${ }^{12} \mathrm{C} /{ }^{13} \mathrm{C}$ ratio determinations. The ${ }^{12} \mathrm{C} /{ }^{13} \mathrm{C}$ ratio was determined from the $(2,0){ }^{13} \mathrm{C}^{12} \mathrm{~N}$ feature at $8004.7 \AA$. All $\log g f$ values were calibrated to fit to the solar spectrum by Kurucz (2005) with solar abundances from Grevesse \& Sauval (2000). In Figure 3 we show several examples of synthetic spectra in the vicinity of ${ }^{12} \mathrm{C}^{14} \mathrm{~N}$ lines.

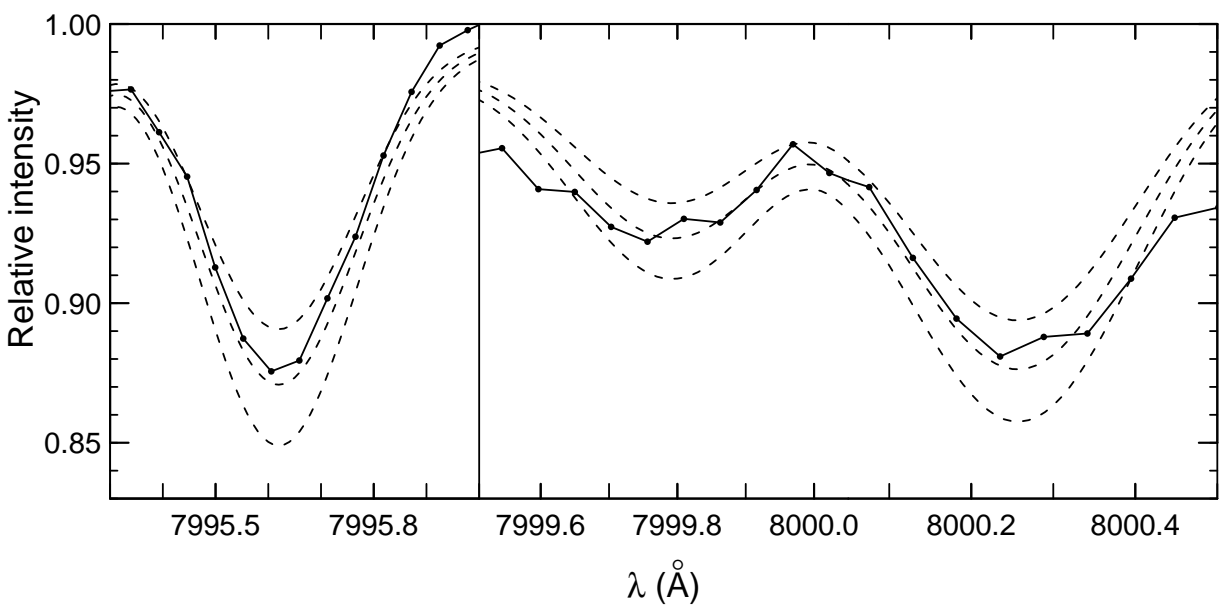

Fig. 3. Synthetic spectrum fits to ${ }^{12} \mathrm{CN}$ lines. The observed spectrum is shown as a solid line. The dashed lines are synthetic spectra with $[\mathrm{N} / \mathrm{Fe}]=0.02,0.12$ and 0.22 dex.

The oxygen abundance was determined from the forbidden [O I] line at $6300.31 \AA$ (Figure 4) with the oscillator strength values for ${ }^{58} \mathrm{Ni}$ and ${ }^{60} \mathrm{Ni}$ from Johansson et al. (2003) and the values $\log g f=-9.917$ obtained by fitting to the solar spectrum (Kurucz 2005) and $\log A_{\odot}=8.83$ taken from Grevesse \& Sauval (2000).

The abundance of Na I was estimated using the line $5148.84 \AA$ which due to rotational broadening is blended by Ni I line at $5148.66 \AA$. These two lines are distinct in the Sun, so we were able to calibrate their $\log g f$ values using the solar spectrum. However, the sodium abundance value in our study is affected by uncertainty of the nickel abundance determination, originating from the Equivalent Widths method. Fortunately, the line-to-line scatter of $[\mathrm{Ni} / \mathrm{H}]$ determination from 24 lines of $\mathrm{Ni}$ I was as small as 0.04 dex.

For the evaluation of $\mathrm{Zr}$ I abundance the lines at $5385.13 \AA, 6127.48 \AA$ and $6134.57 \AA$ were used. Evaluation of Y II abundance (Figure 5) was based on $5402.78 \AA$, Pr II on $5259.72 \AA$ La II on $6390.48 \AA$, Ce II on $5274.22 \AA$ and $6043.38 \AA$ and Nd II on $5276.86 \AA$ lines.

The abundance of Eu II was determined from the $6645.10 \AA$ line (Figure 6 ). The hyperfine structure of Eu II was taken into account when calculating the synthetic spectrum. The wavelength, excitation energy and total $\log g f=0.12$ were taken 


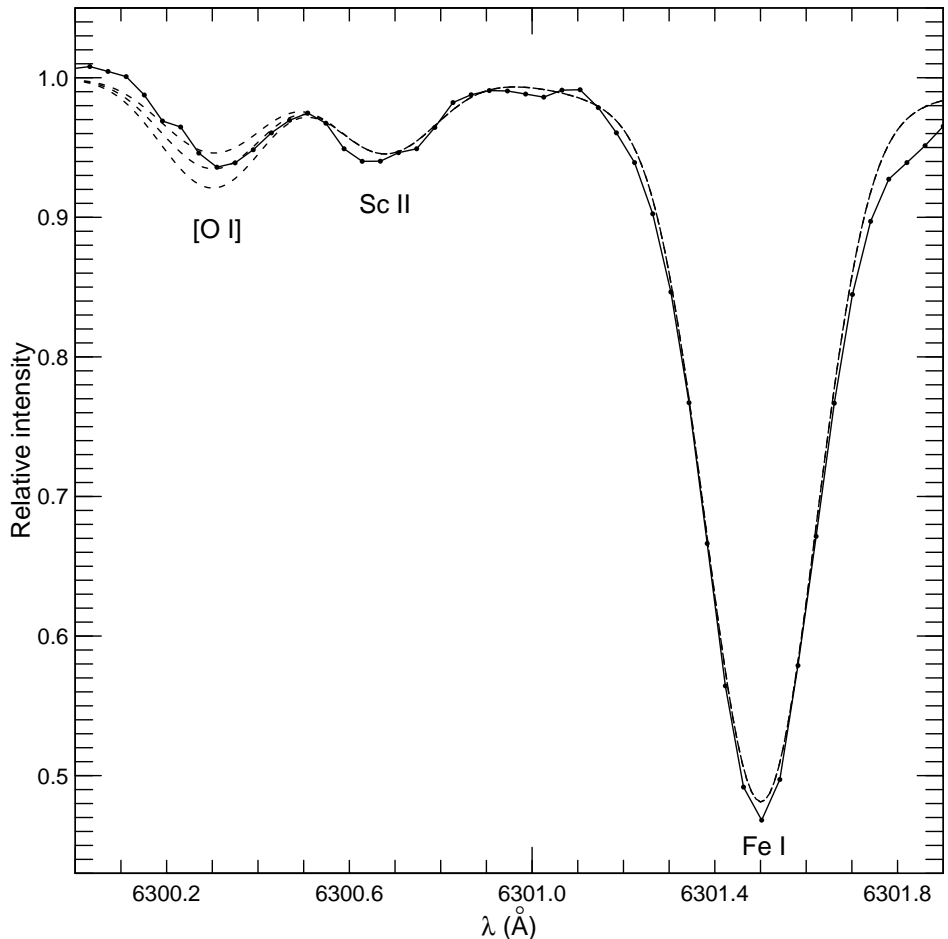

Fig. 4. Synthetic spectrum fit to forbidden [O I] line at $6300 \AA$. The observed spectrum is shown as a solid line. The dashed lines are synthetic spectra with $[\mathrm{O} / \mathrm{Fe}]=$ $-0.14,-0.04$ and 0.06 dex.

from Lawler et al. (2001), the isotopic meteoritic fractions of ${ }^{151} \mathrm{Eu}, 47.77 \%$, and ${ }^{153} \mathrm{Eu}, 52.23 \%$ and isotopic shifts were taken from Biehl (1976).

Due to the rotation of RS CVn stars, their spectral lines are broadened, so it is important to use a correct value of $v \sin i$ in the synthetic spectrum calculations. We used $v \sin i=6.7 \mathrm{~km} \mathrm{~s}^{-1}$ from Fekel (1997), which fits well the profiles of spectral lines of 29 Dra.

\subsection{Estimation of uncertainties}

The sources of uncertainty were described in detail in Paper I. The sensitivity of the abundance estimates to changes in the atmospheric parameters for the assumed errors $\left( \pm 100 \mathrm{~K}\right.$ for $T_{\text {eff }}, \pm 0.3$ dex for $\log g$ and $\pm 0.3 \mathrm{~km} \mathrm{~s}^{-1}$ for $\left.v_{\mathrm{t}}\right)$ is illustrated in Table 2. It is seen that possible parameter errors do not affect the abundances seriously; the element-to-iron ratios, which we use in our discussion, are even less sensitive.

The scatter of the deduced line abundances $\sigma$, presented in Table 3, gives an estimate of the uncertainty due to the random errors, e.g., in the continuum placement and the line parameters (the mean value of $\sigma$ is $0.05 \mathrm{dex}$ ). Thus the uncertainties in the derived abundances originating from the random errors are close to this value. 


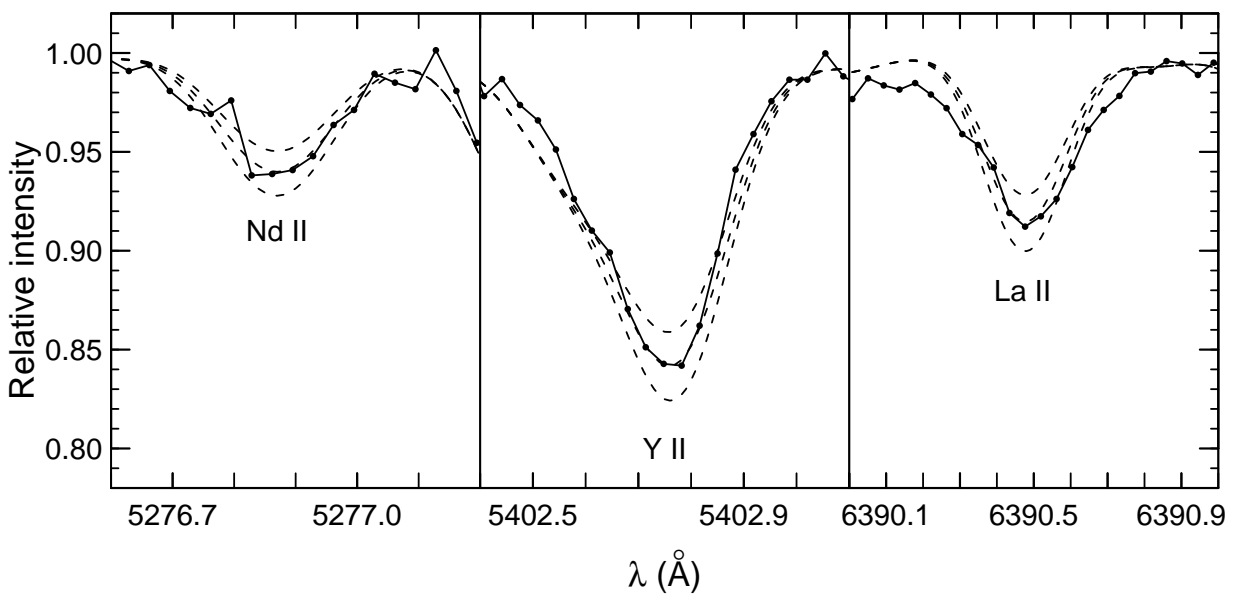

Fig. 5. Synthetic spectrum fit to the Nd II line at $5276.806 \AA$, Y II at $5402.78 \AA$ and La II at $6390.48 \AA$. The observed spectrum is shown as a solid line. The dashed lines are synthetic spectra with $[\mathrm{Nd} / \mathrm{Fe}]=0.08,0.18$ and $0.28 \mathrm{dex},[\mathrm{Y} / \mathrm{Fe}]=0.01,0.11$ and 0.21 dex and $[\mathrm{La} / \mathrm{Fe}]=0.07,0.17$ and 0.27 dex, respectively for Nd II, Y II and La II lines.

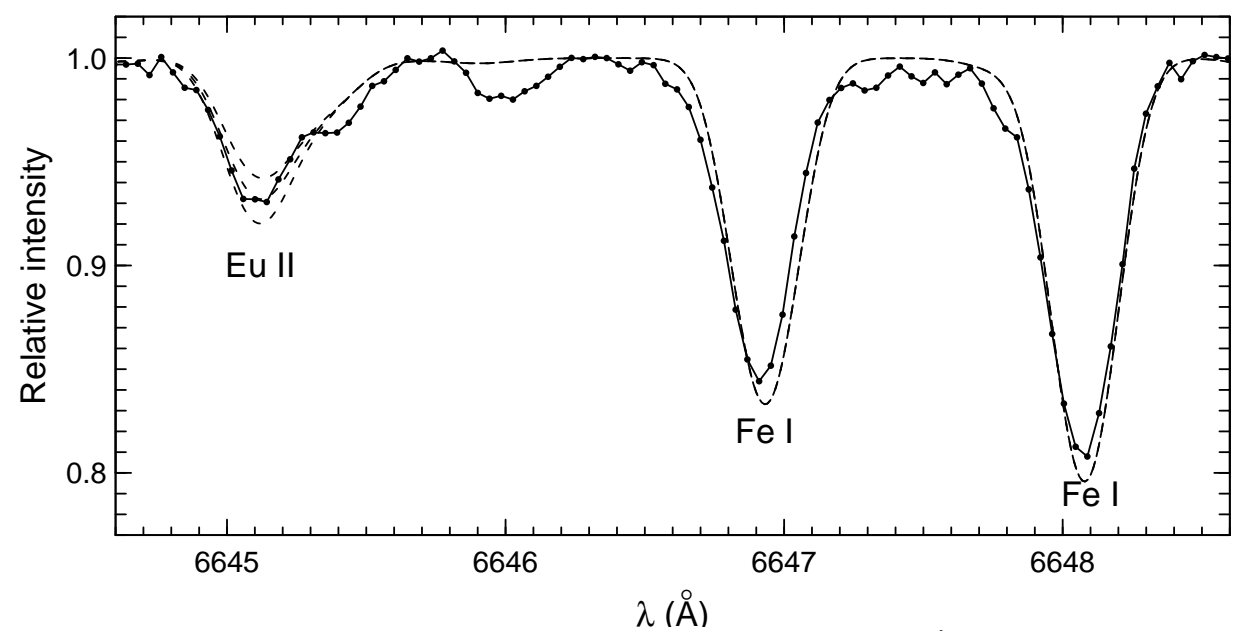

Fig. 6. Synthetic spectrum fit to the Eu II line at $6645.10 \AA$ đं is shown as a solid line. The dashed lines are synthetic spectra with $[\mathrm{Eu} / \mathrm{Fe}]=-0.03$, 0.07 and 0.17 dex.

Since the abundances of $\mathrm{C}, \mathrm{N}$ and $\mathrm{O}$ are bound together by the molecular equilibrium, we have also investigated how an error in one of them typically affects the abundance determination of others.

$\Delta[\mathrm{O} / \mathrm{H}]=0.10$ causes $\Delta[\mathrm{C} / \mathrm{H}]=0.04$ and $\Delta[\mathrm{N} / \mathrm{H}]=0.03 ; \Delta[\mathrm{C} / \mathrm{H}]=0.10$ causes $\Delta[\mathrm{N} / \mathrm{H}]=-0.12$ and $\Delta[\mathrm{O} / \mathrm{H}]=0.02 . \Delta[\mathrm{N} / \mathrm{H}]=0.10$ has no effect on both carbon and oxygen abundances. 
Table 2. Sensitivity of the abundances to changes of the atmospheric parameters. The table entries show the effects on the logarithmic abundance relative to hydrogen, $\Delta[\mathrm{A} / \mathrm{H}]$

\begin{tabular}{|c|c|c|c|c|c|c|c|}
\hline Ion & $\begin{array}{c}\Delta T_{\text {eff }} \\
+100 \mathrm{~K}\end{array}$ & $\begin{array}{c}\Delta \log g \\
+0.3\end{array}$ & $\begin{array}{c}\Delta v_{\mathrm{t}} \\
+0.3 \mathrm{~km} \mathrm{~s}^{-1}\end{array}$ & Ion & $\begin{array}{c}\Delta T_{\text {eff }} \\
+100 \mathrm{~K}\end{array}$ & $\begin{array}{c}\Delta \log g \\
+0.3\end{array}$ & $\begin{array}{c}\Delta v_{\mathrm{t}} \\
+0.3 \mathrm{~km} \mathrm{~s}^{-1}\end{array}$ \\
\hline $\mathrm{C}\left(\mathrm{C}_{2}\right)$ & 0.00 & 0.11 & 0.00 & Fe II & -0.09 & 0.18 & -0.06 \\
\hline $\mathrm{N}(\mathrm{CN})$ & 0.06 & 0.10 & -0.01 & Co I & 0.06 & 0.06 & -0.06 \\
\hline $\mathrm{O}([\mathrm{O} \mathrm{I}])$ & 0.00 & 0.16 & -0.01 & $\mathrm{Ni}$ I & 0.03 & 0.08 & -0.09 \\
\hline $\mathrm{Na} I$ & 0.12 & 0.00 & 0.00 & Y II & 0.00 & 0.13 & -0.04 \\
\hline Si I & -0.03 & 0.07 & -0.04 & Zr I & 0.19 & 0.00 & -0.01 \\
\hline Ca I & 0.09 & -0.01 & -0.09 & La II & 0.01 & 0.24 & 0.08 \\
\hline Sc II & -0.01 & 0.14 & -0.08 & CeII & 0.01 & 0.14 & -0.04 \\
\hline Ti I & 0.14 & 0.01 & -0.06 & Pr II & 0.02 & 0.14 & -0.02 \\
\hline $\mathrm{V}_{\mathrm{I}}$ & 0.16 & 0.01 & -0.08 & Nd II & 0.01 & 0.14 & -0.01 \\
\hline Cr I & 0.08 & -0.01 & -0.07 & Eu II & -0.01 & 0.16 & -0.01 \\
\hline $\mathrm{Fe} I$ & 0.05 & 0.04 & -0.07 & & & & \\
\hline $\mathrm{C} / \mathrm{N}$ & -0.31 & 0.05 & 0.05 & ${ }^{12} \mathrm{C} /{ }^{13} \mathrm{C}$ & 0 & 1 & 0 \\
\hline
\end{tabular}

Table 3. Element abundances relative to hydrogen, $[\mathrm{A} / \mathrm{H}] . \sigma$ is a standard deviation in the mean value due to the line-to-line scatter within the species. $N$ is the number of lines used for the abundance determination.

\begin{tabular}{lrrrlrcc}
\hline Ion & $N$ & {$[\mathrm{~A} / \mathrm{H}]$} & $\sigma$ & Ion & $N$ & {$[\mathrm{~A} / \mathrm{H}]$} & $\sigma$ \\
\hline $\mathrm{C}\left(\mathrm{C}_{2}\right)$ & 1 & -0.34 & - & Fe II & 6 & -0.21 & 0.07 \\
$\mathrm{~N}(\mathrm{CN})$ & 4 & -0.12 & 0.01 & $\mathrm{Co} \mathrm{I}$ & 8 & -0.13 & 0.07 \\
$\mathrm{O}([\mathrm{O} \mathrm{I}])$ & 1 & -0.24 & - & $\mathrm{Ni} \mathrm{I}$ & 24 & -0.22 & 0.04 \\
$\mathrm{Na}$ I & 1 & -0.02 & - & Y II & 1 & -0.09 & - \\
$\mathrm{Si}$ I & 7 & -0.11 & 0.12 & Zr I & 3 & -0.18 & 0.08 \\
$\mathrm{Ca}$ I & 4 & 0.04 & 0.07 & La II & 1 & -0.03 & - \\
$\mathrm{Sc} \mathrm{II}$ & 5 & -0.14 & 0.06 & Ce II & 2 & -0.30 & 0.01 \\
$\mathrm{Ti} \mathrm{I}$ & 18 & -0.12 & 0.06 & Pr II & 1 & -0.24 & - \\
$\mathrm{V} \mathrm{I}$ & 19 & -0.06 & 0.06 & Nd II & 1 & -0.02 & - \\
$\mathrm{Cr}$ I & 7 & -0.10 & 0.07 & Eu II & 1 & -0.13 & - \\
Fe I & 50 & -0.20 & 0.04 & & & & \\
\hline
\end{tabular}

\section{RESULTS AND DISCUSSION}

As a result, for 29 Dra we found the following atmospheric parameters: $T_{\text {eff }}=$ $4720 \mathrm{~K}, \log g=2.5, v_{\mathrm{t}}=1.4 \mathrm{~km} \mathrm{~s}^{-1},[\mathrm{Fe} / \mathrm{H}]=-0.20,[\mathrm{C} / \mathrm{Fe}]=-0.14,[\mathrm{~N} / \mathrm{Fe}]=$ $0.08,[\mathrm{O} / \mathrm{Fe}]=-0.04$, as well as the ratios $\mathrm{C} / \mathrm{N}=2.40$ and ${ }^{12} \mathrm{C} /{ }^{13} \mathrm{C}=16$. The element abundances $[\mathrm{A} / \mathrm{H}]$ and the $\sigma$ values (the line-to-line scatter) are listed in Table 3 and displayed in Figure 7.

The effective temperature and surface gravity values for 29 Dra given in different papers are quite similar. $\mathrm{T}_{\text {eff }}=4700 \mathrm{~K}$ was determined by Randich et al. (1994), $4800 \mathrm{~K}$ by Berdyugina (1994) and Začs et al. (1997) and $4720 \mathrm{~K}$ in the present paper. Our value of $\log g=2.5$ is in agreement with the result of Začs et al. (1997). Randich et al. (1994) have found the value larger by 0.1 dex. From the wings of $\mathrm{MgH}$ lines, Berdyugina (1994) has found $\log g=2.7$.

The $[\mathrm{Fe} / \mathrm{H}]$ value of -0.20 dex determined in the present paper is close to the value of Začs et al., -0.24 dex. A slightly higher value, -0.05 dex, was found by Berdyugina and an exceptionally low value of -0.8 dex by Randich et al. 
In Figure 7, the element to iron ratios for 29 Dra are compared with the results of other investigations. The element to iron ratios for the iron peak, $s$ - and $r$-process elements are solar. Abundances of $\alpha$-elements are enhanced by about 0.1 dex.

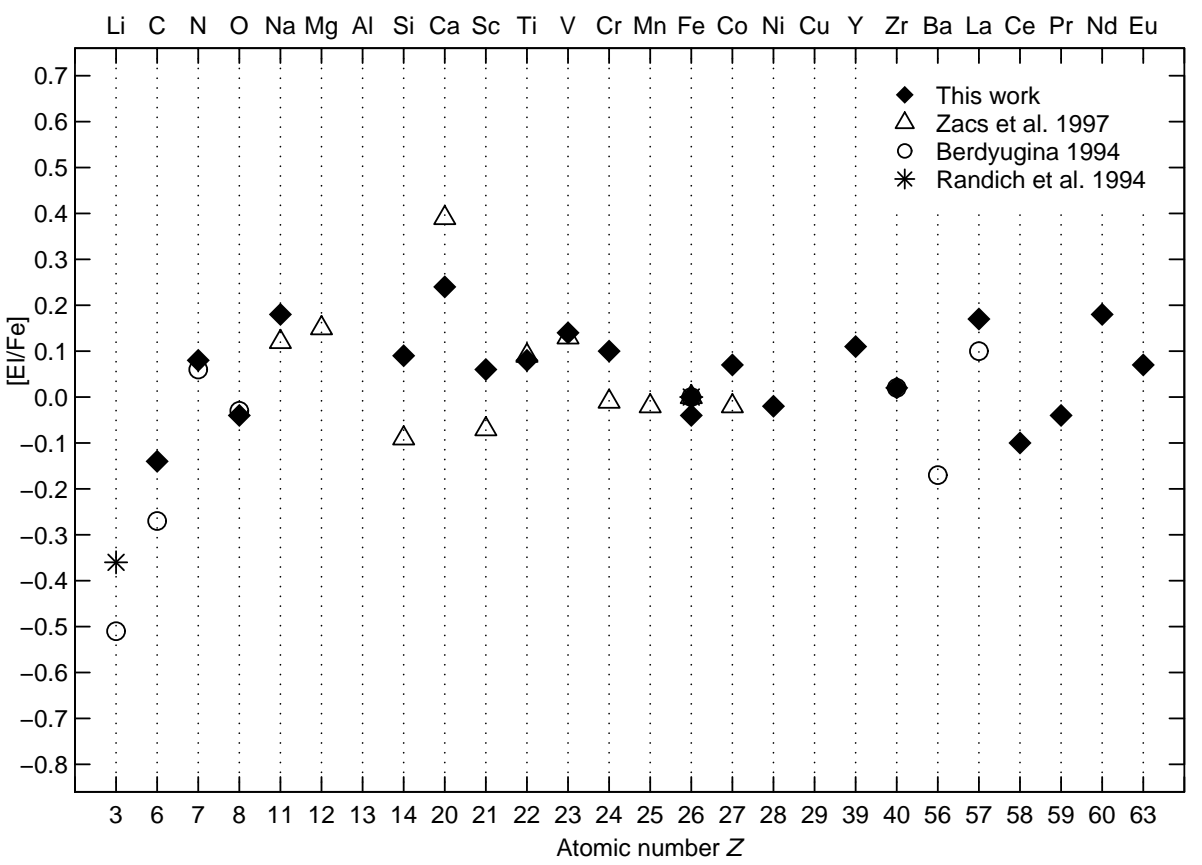

Fig. 7. Element abundances for 29 Dra, as determined in this work (filled diamonds), by Začs et al. (1997, triangles), Berdyugina (1994, circles) and Randich et al. (1994, asterisks).

\subsection{The ratios $C / N$ and ${ }^{12} C /{ }^{13} C$}

The evolutionary sequences in the luminosity versus effective temperature diagram by Girardi et al. (2000) show that 29 Dra with its luminosity $\log \left(L / L_{\odot}\right)=$ 1.44 is a first ascent giant lying slightly below the red giant sequence bump at $\log \left(L / L_{\odot}\right)=1.6$ (Charbonnel \& Lagarde 2010). According to the mentioned model of mixing, carbon and nitrogen abundances in 29 Dra should be altered only by the first dredge-up. The ratio ${ }^{12} \mathrm{C} /{ }^{13} \mathrm{C}$ for the first ascent giants with the mass of 29 Dra $\left(1.2 M_{\odot}\right)$ should be lowered to the value of about 26 (Charbonnel \& Lagarde 2010). However, the ratio found in this paper is 16.

In Figure 8, we compare $\mathrm{C} / \mathrm{N}$ and ${ }^{12} \mathrm{C} /{ }^{13} \mathrm{C}$ ratios of 29 Dra with the theoretical models including extra mixing. The model, called 'cool bottom processing' (CBP), was proposed by Boothroyd \& Sackmann (1999) and the model, called 'thermohaline mixing' (TH), was proposed by Charbonnel \& Lagarde (2010). The position of 29 Dra in Figure 8 indicates that its ratio of carbon isotopes has been altered by extra mixing. The low value of the ${ }^{12} \mathrm{C} /{ }^{13} \mathrm{C}$ ratio was also found in $\lambda$ And (Paper I). 

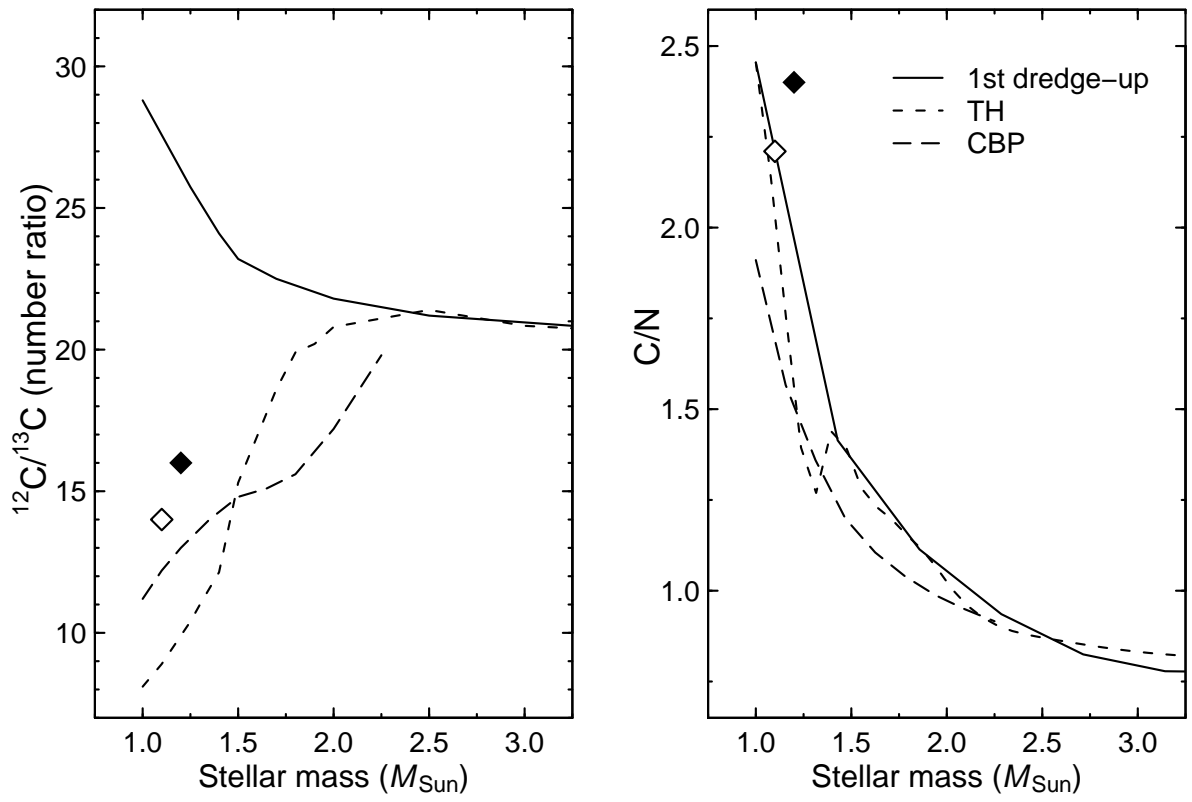

Fig. 8. Comparisons of $\mathrm{C} / \mathrm{N}$ and ${ }^{12} \mathrm{C} /{ }^{13} \mathrm{C}$ ratios in 29 Dra (filled diamonds) and $\lambda$ And (open diamonds, Paper I) with the theoretical predictions explained in the text.

The abundance of lithium is also very sensitive to mixing. During the first dredge-up, for a star of the mass of 29 Dra, Li abundance drops to $\log A(\mathrm{Li})=$ 1.45 (Charbonnel \& Lagarde 2010). However, the available determinations of Li abundance in 29 Dra show lower abundances. Berdyugina (1994) finds $\log A(\mathrm{Li})=$ 0.6 and Randich et al. (1994) find $\log A(\mathrm{Li}) \leq 0$. The low abundance of $\mathrm{Li}$ is compatible with the thermohaline extra-mixing model by Charbonnel \& Lagarde (2010), which predicts the lowering of $\mathrm{Li}$ abundance to the value of about 0.07 dex. The observed Li abundances in $\lambda$ And are even lower (Savanov \& Berdyugina 1994; Randich et al. 1994; Mallik 1998).

Thus, both $\lambda$ And and 29 Dra, investigated by now in our program, give a hint that extra-mixing processes may start acting in these low-mass chromospherically active fast rotating stars slightly earlier than at the bump of the red giant sequence in non-active stars.

ACKNOWLEDGMENTS. This project has been supported by the European Commission through the Baltic Grid II project.

\section{REFERENCES}

Abt H. A., Biggs. E. S. 1972, Bibliography of Stellar Radial Velocities, KPNO, Tucson

Alonso A., Arribas S., Martínez-Roger C. 1999, A\&AS, 140, 261

Berdyugina S. V. 1994, Astronomy Letters, 20, 796

Bidelman W. P. 1954, ApJS, 1, 175

Biehl D. 1976, Diplomarbeit, Christian-Albrechts-Universität Kiel, Institut für Theoretische Physik und Sternwarte 
Boothroyd A. I., Sackman I. J. 1999, ApJ, 510, 232

Charbonnel C., Lagarde N. 2010, A\&A, 522, A10

Fekel F. C. 1997, PASP, 109, 514

Fekel F. C., Simon T. 1985, AJ, 90, 812

Fekel F. C., Henry G. W., Busby M. R., Eitter J. 1993, AJ, 106, 2370

Girardi L., Bressan A., Bertelli G., Chiosi C. 2000, A\&AS, 141, 371

Grevesse N., Sauval A. J. 2000, in Origin of Elements in the Solar System, Implications of Post-1957 Observations, ed. O. Manuel, Kluwer, p. 261

Hakkila J., Myers J. M., Stidham B. J., Hartmann D. H. 1994, AJ, 114, 2043

Hall D. S., Henry G. W., Louth H., Renner T. R., Shore S. N. 1982, IBVS, 2109, 1

Hauck B., Mermilliod M. 1998, A\&AS, 129, 431

Johansson S., Litzen U., Lundberg H., Zhang Z. 2003, ApJ , 584, 107

Kurucz R. L. 2005, New Atlases for Solar Flux, Irradiance, Central Intensity, and Limb Intensity, Mem. SA Ital. Suppl., 8, 189

Lawler J. E., Wickliffe M. E., Den Hartog E. A. 2001, ApJ, 563, 1075

van Leeuwen F. 2007, Hipparcos, the New Reduction of the Raw Data, Astrophysics and Space Science Library, vol. 350

Mallik S. V. 1998, A\&A, 338, 623

Randich S., Giampapa M. S., Pallavicini R. 1994, A\&A, 283, 893

Roman N. G. 1955, ApJS, 2, 195

Savanov I. S., Berdyugina S. V. 1994, Astronomy Letters, 20, 227

Tautvaišienė G., Barisevičius G., Berdyugina S., Chorniy Y., Ilyin I. 2010, Baltic Astronomy, 19, 95 (Paper I)

Začs L., Musaev F. A., Bikmaev I. F., Alksnis O. 1997, A\&AS, 122, 31

Zboril M., Messina S. 2009, AN, 330, 377 